\title{
Earthquake Damage Predicting System of Songyuan Based on GIS
}

\author{
Su Zhenjiang \\ Institute of Disaster prevention \\ The GIS Association of Institution of Disaster Prevention \\ Beijing, China \\ e-mail: 75672946@qq.com \\ Huang Meng* \\ Institute of Disaster prevention \\ The GIS Association of Institution of Disaster Prevention \\ Beijing, China \\ e-mail: 398727732@qq.com
}

Xie Shaohui

Institute of Disaster prevention

The GIS Association of Institution of Disaster Prevention

Beijing, China

e-mail: 1004120572@qq.com

\author{
Zhang Dian \\ Institute of Disaster prevention \\ The GIS Association of Institution of Disaster Prevention \\ Beijing, China \\ e-mail:774847587@qq.com \\ Wang Zhe \\ Institute of Disaster prevention \\ The GIS Association of Institution of Disaster Prevention \\ Beijing, China \\ e-mail: 1015382294@qq.com
}

\begin{abstract}
With the rapid development of cities, the research on urban seismic damage prediction should have a breakthrough based on the modern technology. The paper takes Songyuan of Jilin Province as the research object, and creates a spatial database by field data and Baidu maps. We simulate the earthquake by using the seismic intensity algorithm of the point source and the line source, use the spatial analysis method of PHP+GIS to simulate the influence at different earthquake levels on the urban buildings in Songyuan. At the same time, PHP, HTML and dynamic cutting technique were used to evaluate the disaster in 10 minutes after the earthquake. This system will provide technical support for the prevention of earthquake damage in Songyuan.
\end{abstract}

Keywords-Earthquake Damage Prediction; Songyuan; Baidu Maps API; Rapid Assessment

\section{INTRODUCTION}

With the rapid development of Chinese economy and the urbanization process, more and more attention is supposed to be put on the potential threat of earthquakes to people's lives and property. If we can use GIS technology to simulate the loss may be caused by the earthquake to the city before the disaster, and estimate the severe disaster area to shrink the scope of rescue, it will provide the management decisionmaking institutions with reliable technical support. Therefore, it is very meaningful to develop a GIS-based earthquake seismic hazard prediction system.

GIS technology, with its intuitive characteristics, is widely used in various fields. For example, in 2009, Professor Yuan Longbo of the School of Civil and Hydraulic Engineering of Dalian University of Technology made an Earthquake predicting system of urban lifeline based on GIS, which made a rational analysis of the influence of urban pipe network connectivity and damage on pipe network, but it didn't comprehensively discuss the influence of pipeline network on urban buildings. In 2012, Zhao Qi, a director of the Structural Engineering and Disaster Prevention Research Institute of Tongji University, made a Study on Application of High Resolution Remote Sensing Images in Rapid Prediction of Earthquake Disaster in Urban Area. This technology adapts to the needs of large-scale regional earthquake damage prediction work, but because remote sensing satellites cannot provide real-time image, the project cannot meet the requirements of post-earthquake rapid assessment. In 2013, Wang Dongming, a researcher at the China Earthquake Prevention Center, made a Research on Urban Earthquake Damage Prediction Virtual Simulation System. Professor Wang adopted the city simulation based on VR technology, but the whole development cost is high, which is not conducive to promotion. In 2016, Xiao Xinghui, a teacher of China Ocean University, made a Prediction Model of Urban Bridge Seismic Damage Based on Least Squares Support Vector Machine, which is mainly aimed at the study of urban bridges, but didn't study the seismic modeling of urban buildings.

To summary, although many researchers have made different directions for urban earthquake damage prediction, but for the rapid assessment of urban construction disaster is still left blank. Therefore, the paper, through the study on earthquake damage prediction and the feasibility of software implementation, has developed an earthquake intensity model software aimed at Songyuan, Jilin Province, and it also is a platform for analysis that can be combined with multiple factors to give disaster assessment and rescue information. 


\section{SySTEM DESIGN}

\section{A. Design of Systematic Framework}

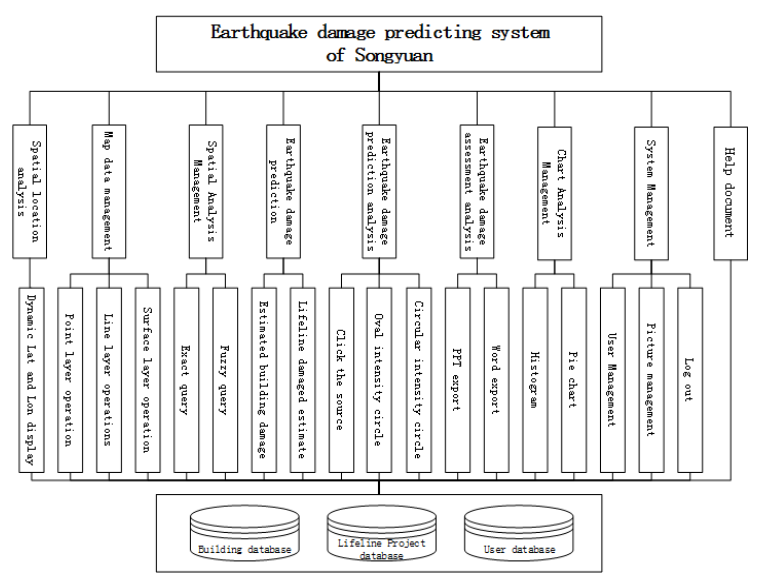

Figure 1. Functional structure diagram

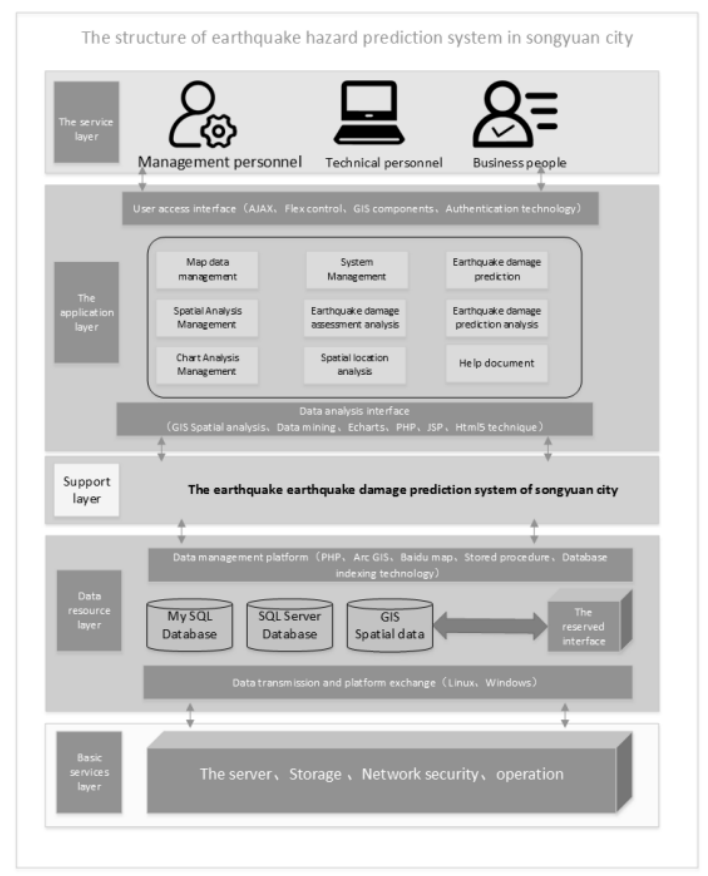

Figure 2. Technical flow chart

\section{B. Database}

The correctness of the prediction results of the earthquake predicting system in Songyuan depends on the reliability of the data source. This project lasted more than a year, we carried out comprehensive field survey and data collection of more than 3000 buildings located at Ningjiang district, Fuyu City, Qian'an County, Changling County and Ross Mongolian Autonomous County in Songyuan, which finally collected about 120 thousand complete messages.
The coordinate system in map is Beijing 54 coordinate system, and the coordinates of building are SHP format data through the field research. The map component is used Baidu maps interface. Because its own coordinates, we need a coordinate conversion. There are many methods for coordinate conversion, and this system uses recursive callback method requesting API for batch processing of coordinate data. Many articles are described in detail about the method, so we don't go into details.

Another place to be pretreated is the vulnerability of building structures to different seismic grades. For this point, the paper draws on the study of the seismic performance of buildings by Yi Qian researcher, carries out the prediction examples based on the damage of Shenyang and Wuhan city, combined with the data of the existing foundation, and uses the vulnerability of building structures to earthquake disaster prediction science describing failure degree of building and loss of the city under different intensity earthquake damage. The specific algorithm is as follows:

Vulnerability of the building structure is the probability or possibility of a certain degree of damage occurring under the determined earthquake intensity. China earthquake intensity table to the building's damage level is divided into basic intact, minor damage, moderate damage, serious damage and destruction of five grades. The researchers divided our buildings into four categories according to the vulnerability level. Class A mainly includes multi-storey steel and reinforced concrete structures, and the seismic capacity is the best. Class B mainly includes brick structure, industrial building and the seismic capacity is inferior to Class A; Class C mainly includes without formal design, open brick structure; D class for the soil structure, is the worst type of earthquake resistance.

$$
\mathrm{VID}=\frac{1}{5} \sum_{I=6}^{10} \sum_{I=1}^{5} P[D \mathrm{j} \mid \mathrm{I}] \lambda_{\mathrm{j}}
$$

In the formula, VID is the structural vulnerability index, it is the seismic damage matrix of building, I means the seismic intensity, $\mathrm{Dj}$ means the damage level of the house, it is the damage rate of the house when the $\mathrm{j}$ damage occurs. The smaller the vulnerability index is, the better the seismic capacity of structure is, and the smaller the earthquake loss is. This project combines the historical seismic damage statistics method and test method, through the collection of buildings under different intensities of damage degree of seismic data in recent years, the analysis of the vulnerability of different types of buildings, combined with the test method to simulate the specific building and the corresponding experimental results and got the intensity of vulnerability index, then fitting out the fragility curves of all kinds of buildings. For example, the project survey in Ningjiang district of Songyuan city is a high-rise concrete building, according to the fragility curves of the building. The building is estimated at six level showed a good intensity, showing good intensity at level seven, damaged in eight level intensity, in nine levels of intensity is moderate 
damage, severe in the ten stage damage intensity. Part of the field of the building database is shown in figure 3 :

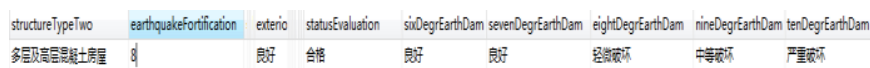

Figure 3. Schematic diagram of database vulnerability

\section{Database Design}

Database plays an important role in the system platform, so it is very important to design a reasonable database structure. The population, economic and geographical data from different data source formats such as TXT text format, EXCLE format, and SHP format, we need to provide for different kinds of data sources in the database data entry interface. At the same time, when the database is built, the data redundancy and the feasibility of the later maintenance are also need to be considered. Through several design schemes, the relational database scheme with the building number as the unique identifier and the multi table association is adopted.

TABLE I. THE MAIN FIELD AND DESCRIPTION

\begin{tabular}{|c|c|c|}
\hline $\begin{array}{l}\text { Field } \\
\text { table }\end{array}$ & Field Name & explanation \\
\hline 1 & buildingNumber & $\begin{array}{l}\text { Primary key column, used for field } \\
\text { matching, multi-table joint query }\end{array}$ \\
\hline 2 & $\begin{array}{l}\mathrm{X} \\
\mathrm{Y}\end{array}$ & $\begin{array}{l}\text { actual coordinates, through the actual } \\
\text { investigation }\end{array}$ \\
\hline 3 & $\begin{array}{l}\text { baidu_X } \\
\text { baidu_Y }\end{array}$ & $\begin{array}{l}\text { Baidu coordinates, through the actual } \\
\text { coordinates of the building } \\
\text { coordinates obtained after conversion }\end{array}$ \\
\hline 4 & propertyType & $\begin{array}{l}\text { Building Type, used to screen } \\
\text { different types of buildings }\end{array}$ \\
\hline 5 & nameOfHous & Head of household name \\
\hline 6 & constructionAge & Building age \\
\hline 7 & $\begin{array}{l}\text { sixDegrEarthDam } \\
\text { sevenDegrEarthDam } \\
\text { eightDegrEarthDam } \\
\text { nineDegrEarthDam } \\
\text { tenDegrEarthDam }\end{array}$ & $\begin{array}{l}\text { Building Vulnerability Level, } \\
\text { Damage degree of housing at different } \\
\text { intensity levels }\end{array}$ \\
\hline
\end{tabular}

This project implements the data import interface for different data sources, and add the relevant attribute information according to the building number to dynamically. Taking into account the late housing information replacement, this project provides an interface for dynamically modifying updated data. With this kind of interface, all data can be updated in real time.

\section{STYLING SYSTEM IMPLEMENTATION}

This paper is based on the point set and line set data from Songyuan city survey, using Baidu map to display the building information and lifeline engineering in Songyuan. The seismic intensity model of point source and line source is used to simulate the earthquake, and the earthquake disaster and building damage are evaluated automatically. User can export the result to the document and export the relevant Word document according to the requirement.
Here we will make a preliminary demonstration of the key functional algorithm ideas:

\section{A. Elliptic intensity attenuation method}

In the work of seismic safety evaluation, earthquake zoning and post-earthquake rapid assessment, as an important indicator of earthquake motion, the attenuation relation and law of earthquake intensity have obvious regionality. Because of the typical plain landform of Songyuan city and a lot of very mature elliptical intensity attenuation research, the paper adopts the intensity attenuation relation of line source earthquake in North China proposed by Sha Haijun as the attenuation model of Songyuan intensity.

$$
\begin{aligned}
& I_{1}=2.033+2.010 M-(0.923+0.151 M) \ln \left(R^{\prime}+27.035\right) \\
& I_{\mathrm{s}}=0.064+1.949 M-(0.433+0.169 M) \ln \left(R^{\prime}+13.073\right)
\end{aligned}
$$

In this formula, the variable I represents the intensity, the variable $M$ means the magnitude, and $R$ represents the epicenter distance $(\mathrm{km})$, the lower corner of the standard $1, \mathrm{~s}$ represents the long and short axis. The difficulty of this project is the combination of elliptical intensity attenuation method and Baidu map to truly achieve the simulation of ellipse intensity on map. For this reason, we abstracted it as a mathematical model to achieve. $\mathrm{F}(\mathrm{X}, \mathrm{Q}, \mathrm{R}, \mathrm{Y}, \mathrm{Z})=0$ is a set of points, $\mathrm{F}$ is the input set, $\mathrm{X}$ is the coordinate point of the focal position, $\mathrm{Q}$ is the strong earthquake action, $\mathrm{R}$ is the epicentral distance, $\mathrm{Y}$ is the deflection angle under the action of the fault zone, $\mathrm{Z}$ is the output set. Then the model is rewritten into a computer language and implemented with a class, and the coordinates of all points on the intensity map are obtained.

Simulation of a 7.5 earthquake occurred, according to the long and short axis intensity attenuation algorithm effect shown in Figure 4.

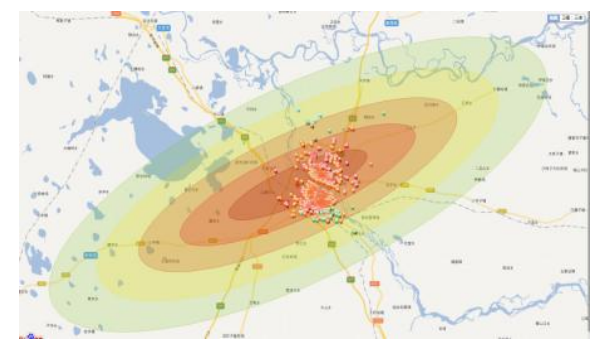

Figure 4. Longitudinal axis elliptical intensity attenuation method

In contrast, the circular intensity attenuation method is relatively simple to implement based on the ellipse. The circular model assumes that the seismic source is a point source, ignoring the effect of seismic fault rupture on ground motion, and the seismic intensity decreases with increasing epicentral distance. As Matsubara is located in the plains, according to the empirical formula of earthquake attenuation, $\mathrm{I}=\mathrm{M}+2$ where $\mathrm{I}$ is the intensity of the earthquake and $\mathrm{M}$ is the magnitude. In general, the higher the intensity of attenuation, the faster the decay of 10 degrees to 9 degrees 
after $5 \mathrm{~km}$ or so, followed by 9 degrees to 8 degrees after $8 \mathrm{~km}, 8$ degrees to 7 degrees after $20 \mathrm{~km}, 7$ degrees to 6 degrees to go through $40 \mathrm{~km}$ or so. According to this model, the intensity attenuation diagram of an earthquake can be dynamically generated by controlling the radius of the circular intensity circle at all levels.

Simulation of the occurrence of a 7 earthquake and according to the disaster situation will be severely affected buildings automatically grouped the effect shown in Figure 5.

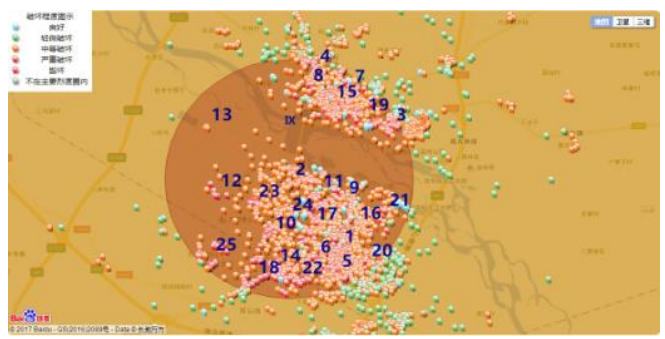

Figure 5. Circular intensity attenuation method

In addition, our system has also realized the function of earthquake damage prediction for lifeline engineering, and the multi layer analysis with the overlay of the building layer. The effect is shown in Figure 6.

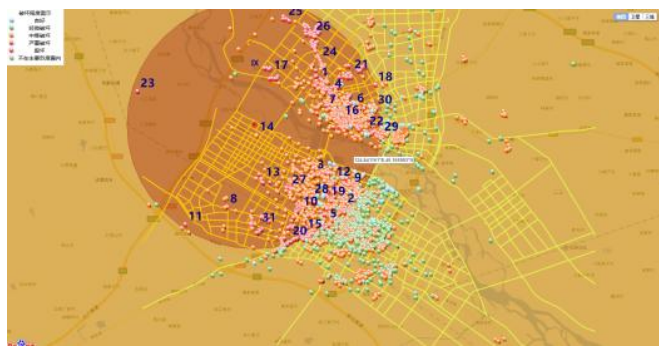

Figure 6. Damage prediction for lifeline engineering

\section{B. Document export}

Document export is a key part of the evaluation system, the user can then manipulate the system to obtain automatically generated documents and data from the composition of the document, which to a large extent save the time to artificially export data. This project uses the PHPWord class library to complete the requirements for generating documents and formats. And designs the delay module by several dynamic statistics, combined with H5 latest technology dynamic capture window real-time capture analysis results, finally can be saved in the cache folder.

Because the ThinkPHP framework does not provide support for the direct operation of Microsoft word and PowerPoint, then we change our mind in the Windows environment. This project starts by modifying the PHP configuration file php.ini to start the support of PHP zip, thus gaining the PHPWord class library 0.6.2-1 version, and extending the support for MS Word and PowerPoint. Because the PHPWord class library is a class library edited abroad, there still exists the problem of Chinese garbled code. It will translate the input text into utf8_ encode code. That is to say, using GBK, GB2312 or utf8 code will appear garbled situation, so we use utf8 encoding. At the same time, we find the utf8_encode transcoding in all the methods in the class library, delete them, and use iconv to encode the GBK or GBK2312 code, which can solve the problem better.

There are 5 pictures produced in the Word document, namely the intensity circle map of the earthquake area, the distribution map of the destruction of the houses in the earthquake area, the pie charts of the houses damaged in the earthquake area, the column of the destruction of the houses in the earthquake area and the architectural situation in the city. Each picture is waiting for the page finishes loading through the delay module, real-time dynamic crawl on the window with screenshots of components, and then gain access to the data stream to the interception time to distinguish cached in the corresponding folder. When exporting, we read the cache file, and we insert the picture through the function called addImage. The module can quickly export the electronic documents of damaged buildings after earthquake simulation, and reduce the occupation of computer memory resources by using cache, which has the characteristics of flexibility and portability.

The seismic damage prediction results of Figure 4 are derived by using Word, which is shown in Figure 7:

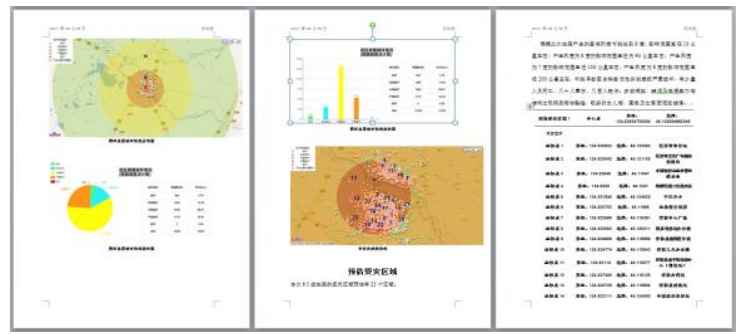

Figure 7. Export the document part of the schematic

\section{CONCLUSION}

This paper is based on the theory of earthquake damage prediction for the terrain of Songyuan, cleverly combines a variety of methods such as seismic intensity algorithm for point source and line source, PHP + GIS spatial analysis method, PHP + HTML + dynamic cutting Technology, to apply GIS theory to the actual development needs. It's different from the previous urban earthquake damage prediction system, and the system data sources are more comprehensive and reliable. In the calculation of data processing in this system, the results have been collated in the form of presenting qualified documents, which is very convenient to the user's operation. The system also has room for upgrading, and it is expected to continue to increase the function module of casualties statistics, so as to make the system function more perfect.

The implementation of the project is based on its rapid exporting of derived products within 10 minutes, which make up for the current earthquake industry in the post earthquake rapid assessment of the blank. The system provides a powerful scientific basis for improving the ability 
of urban earthquake prevention and disaster reduction by revealing the weak links of urban building earthquake. At the same time, it also provides some technical support for earthquake damage prediction in Songyuan.

\section{ACKNOWLEDGMENTS}

This work was supported by the Special Fund of Fundamental Scientific Research Business Expense for Higher School of Central Government (No. ZY20160106).

\section{REFERENCES}

[1] Yuan Yongbo, Bai Guangbin, Zhang Mingyuan. GIS based prediction system for earthquake damage of urban lifelines Liaoning University of Technology and Technology (Natural Science Edition, December
2010) Technology based on GIS in Changchun City earthquake damage prediction technology 2009-04

[2] Zhao Qi, Zhai Yong-mei, Li Tiezheng Application of high-resolution remote sensing image in urban rapid earthquake damage prediction [J]. Software Journal, 2012, 02-0072-05

[3] Wang Dongming, Study on virtual simulation system of urban earthquake damage prediction [J]. China Earthquake Disaster Prevention Center, 2013, 01-0098-05

[4] Zhang Yu, Kang Jianhong, Wei Meixuan, Li Na, Yang Qing, Study on prediction model of earthquake damage of urban bridge based on least squares support vector machine. Geophysical and geochemical exploration, 2014, 38 (5); 03-0124-08

[5] Wu Qing, Gao Mengtan. Study on historical earthquake magnitude and epicenter using elliptical intensity attenuation relationship [J]. Institute of Geophysics, China Earthquake Administration, 2015, 12 : 76-79. 